\title{
Diarrheagenic Escherichia Coli and probiotic activity against foodborne pathogens:A brief review
}

\begin{abstract}
Diarrheagenic Escherichia coli (DEC) represents the most common bacterial pathogen worldwide and is often involved in intestinal illnesses caused by food borne pathogens. DECgenerallycause auto limited watery diarrhea that in some cases can progress to more severe disease, what is related to a range of virulence factors. Probiotic bacteria naturally live in microflora and confer health benefits to the host. Lactobacillus and Bifidobacterium species are the most commonly used as probiotics in research and studies highlighted probiotic activity against DEC and other food borne pathogens by these strains. Studies show that probiotic strains have antimicrobial properties, inhibiting virulence in vitro and in vivo and providing immunomodulation. However, the role of probiotics against pathogenic $E$. coli is still little known and further studies are needed to understand probiotic mechanisms against DEC
\end{abstract}

Keywords: Diarrheagenic Escherichia coli, Food borne pathogens, probiotic activity, Lactobacillus, Bifidobacterium
Volume 7 Issue 5 - 2017

\author{
Andréa Fonseca Ferreira,Wania F Pereira \\ Manfro, Ana Claudia de Paula Rosa \\ Departamento de Microbiologia, Universidade do Estado do Rio \\ de Janeiro, Brazil
}

\begin{abstract}
Correspondence: Ana Claudia de Paula Rosa, Departamento de Microbiologia, Imunologia e Parasitologia (DMIP), Faculdade de Ciências Médicas (FCM), Universidade do Estado do Rio de Janeiro, Boulevard 28 de Setembro, 87 - fundos, $3^{\circ}$ andar.Vila Isabel, Rio de Janeiro, RJ - Brasil, CEP: 2055I-030, Tel + 55 (2I) 2868-8280, Fax +55 (2I) 2868-8376,

Email anarosa2004@gmail.com
\end{abstract}

Received: June 19,2017 | Published: September 05, 2017
Abreviations: DEC, diarrheagenic Escherichia coli; ETEC, enterotoxigenic E. coli; EHEC, Enterohemorrhagic E. coli; STEC, shiga-toxin E. coli; EPEC, Enteropathogenic E. coli; EIEC, Enteroinvasive E. coli; EAEC, Enteroaggregative E. coli; DAEC, Diffusely adherent $E$. coli

\section{Introduction}

Foodborne pathogens cause several diseases, most associated with intestinal symptoms. Bacterial enteropathogens species are highlighted as the cause of several gastrointestinal illnesses, which includes pathogenic Escherichia coli. Diarrheagenic Escherichia coli (DEC) represents the most common bacterial pathogen worldwide.

E. coli strains are naturally commensal bacteria that live in intestinal tract of humans and other mammals. Some strains are pathogenic and can cause disease, especially intestinal.$^{2}$. Diarrheagenic E. coli strains are classified into six categories, based in clinical syndromes, symptoms, epidemiology, O:H serotypes, virulence factors and interaction with in vitro cultivated cells. ${ }^{3}$ All enteropathogenic $E$. coli types cause generally watery diarrhea as a common symptom. Most episodes of intestinal dysfunction are auto limited and solved in a few days, but some rare cases can progress to more severe disease..$^{2,3}$

Probiotics are live nonpathogenic microorganisms that confer health benefits. ${ }^{4}$ Some bacterial probiotic strains provide protection from pathogens to their human hosts, naturally living in the urogenital and gastrointestinal microflora and being usually found in fermented food, like dairy products. ${ }^{5,6}$

\section{Diarrheagenic Escherichia coli}

Enterotoxigenic E. coli (ETEC) strains are enterotoxic (produce heat-stable and heat-labile toxins) and non-invasive of the small intestine, highly associated to traveler's disease.? Enterohemorrhagic E. coli (EHEC) is a common as a food or waterborne pathogen in developing countries. High importance is given to O157:H7 serotype, isolated from stool samples diarrhea outbreaks caused by E. coli. This serotype is related to Shiga-toxin production (Shiga-toxin E. coli - STEC) and destruction of epithelium, which are associated with the development of severe complications, such hemorrhagic colitis and hemolytic uremic syndrome. ${ }^{8}$ Despite being close related to EHEC, enteropathogenic E. coli (EPEC) strains are non-toxin-producing bacteria associated with acute and protracted diarrhea in children in developing countries. ${ }^{3,9}$ Enteroinvasive $E$. coli(EIEC) strains arehighly invasive and non-producing toxins. The pathogenic mechanism is similar in many aspects to the syndrome caused by Shigellacausing severe damage in the intestinal wall by cell destruction. ${ }^{10}$ Enteroaggregative E. coli (EAEC) is defined as $E$. coli that do not produces the heat-stable or heat-labile toxins of ETEC and manifest a characteristic aggregative ('stacked brick') pattern of adherence to HEp2-cells in culture. This pathotype is related to persistent diarrhea in children.$^{1,11}$. Diffusely adherent $E$. coli (DAEC) refers to any HEp-2-adherent $E$. coli strain that form diffuse pattern of adhesion. Its role in diarrhea development is not clearly known. ${ }^{2}$

\section{Probiotic activity against DEC and Other Foodborne Pathogens}

Lactobacillus and Bifidobacterium species are the most commonly used as probiotics in research and some of them are addressed below. Studies highlighted these probiotic strains activities against DEC and other foodborne pathogens, such as reduction of biofilm development, inhibition of adhesion and injury of in vitro cultivated cells, preventing diarrhea on in vivo models and immunomodulation.

L. acidophilus was capable to reduce E. coli O157:H7 (EHEC) and E. coli O127:H6 (EPEC) adhesion to T84 cells . ${ }^{9}$ and Listeria monocytogenes biofilm formation, which was susceptible to probiotic cultures.$^{12}$. Compared to antibiotic use, L. acidophilus showed great inhibition of Campylobacter jejuni, E. coli $\mathrm{O} 157$ and S. typhimurium strains. Furthermore, in vivo, L. acidophillus reduced or prevented diarrhea in albino rats co-infected with Campylobacter jejuni. ${ }^{13}$ Synergistically to L. plantarum, L. acidophilus showed better dose-dependent antimicrobial activity against multidrug resistant EAEC when compared to monostrain condition, also being efficient to decrease EAEC counts in infected mice feces.$^{14}$ L. plantarum showed antimicrobial activity against several enteropathogenic bacteria, including Listeria monocytogenes, Shigella sonnei, Salmonella 
Typhimurium, Staphylococcus aureus and E. coli O157:H7. Also, L. plantarum promoted reduction of cell adhesion by E. coli $\mathrm{O} 157: \mathrm{H} 7, S$. aureus and Salmonella in Caco-2 cells and increased expression of an anti-inflammatory cytokine IL-10 and decreased expression of proinflammatory cytokines IL-6, IL-8, and TNF- $\alpha$ by the cells when co-cultivated with Salmonella. ${ }^{15}$ Otherwise, in a previous study $L$. casei and $L$. plantarum showed no effect on binding or invasion of $E$. coli $\mathrm{O} 157: \mathrm{H} 7$ in Caco-2 cells, meanwhile L. rhamnosus showed outstanding potential for adhering to the cells and inhibiting $E$. coli invasion. ${ }^{16}$ As observed with $L$. acidophilus, L. rhamnosus also promoted a dose-dependent reduction in the adhesion of $E$. coli $\mathrm{O} 157: \mathrm{H} 7$ to HEp-2 and T84 cells, decreasing foci of cytoskeletal rearrangement numbers, which are referred to attaching and effacing lesions ${ }^{9}$ and Listeria monocytogenes biofilm formation. ${ }^{12}$ Similar results were found in another study, where polarized MDCK-I and T84 cells pretreated with $L$. rhamnosus reduced morphological changes and decreased attaching-effacing lesions induced by E. coli $\mathrm{O} 157: \mathrm{H} 7$ infection. ${ }^{17}$ In addition, $L$. casei promotes specific immune responses against $E$. coli $\mathrm{O} 157: \mathrm{H} 7$ cytotoxins and enhances the elimination of bacteria from the intestinal tract in infected rabbits. ${ }^{18} \mathrm{~L}$. casei can also be a vector of heterologous EPEC antigens, stimulating the immune system to produce antibodies that inhibit EPEC adherence to HEp-2 cells. $^{19}$

There are fewer studies about Bifidobacterium spp. probiotic properties against bacterial enteropathogens when compared to lactobacilli and this involves a range of multispecies evaluation. $B$. breve is capable to protect murine intestinal infection caused by $E$. coli $\mathrm{O} 157: \mathrm{H7}$. The strain modifies $\mathrm{pH}$ due to production of high levels of acetic acid and inhibits Shiga-toxin production by STEC growth in vitro. ${ }^{20} \mathrm{~B}$. lactis and $\mathrm{B}$. longum showed antimicrobial activity against Shigella sonnei and have protective properties against uropathogenic E. coli. ${ }^{21} \mathrm{~A}$ B. lactis strain provided protection to Salmonella infection in mice, increasing survival rate and reducing bacterial translocation to spleen and liver. ${ }^{22} B$. animalis and $B$. lactis also promote immunomodulation of mucosa, increasing the number of IgA+ cells. ${ }^{23}$ Increasing in cytokine production and natural killer cells activity can be provided by $B$. animalis and lactobacilli. This property is related to yoghurt consumption, which is a font of multispecies probiotics. ${ }^{6}$

\section{Conclusion}

Probiotic bacteria have potential therapeutic effect and can be used as an alternative treatment in gastrointestinal illnesses. Different strains of the most studied probiotic species promote activity against a range of enteropathogenic bacteria, inhibiting growth, virulence in vitro and in vivo, competing and providing immunological response, especially when they act synergistically. The role of probiotics against pathogenic $E$. coli is still little known. Thus, further studies are needed to understand the role of probiotics activity against DEC.

\section{Aknowledgments}

This work was supported by a grant from FAPERJ, CNPq, CAPES and SR-2/UERJ.

\section{Conflicts of interest}

The authors declare there is no conflict of interest.

\section{Funding}

None.

\section{References}

1. Boisen, N, Krogfelt KA, Nataro JP. Enteroaggregative Escherichia coli. In: Michael Donnenberg editor. Escherichia coli: Pathotypes and Principles of Pathogenesis. 2nd edn, Chapter 8, Academic Press, London, UK, 2013. p. 247-273.

2. Kaper JB, Nataro JP, Mobley HL. Pathogenic Escherichia coli. Nat Rev Microbiol. 2004;2(2):123-140.

3. Nataro JP, Kaper JB. Diarrheagenic Escherichia coli. Clin Microbiol Rev. 1998;11(1):142-201.

4. Reid G, Jass J, Sebulsky MT, et al. Potential uses of probiotics in clinical practice. Clin Microbiol Rev. 2003;16(4):658-672.

5. Liévin-Le Moal V, Servin AL. Anti-infective activities of lactobacillus strains in the human intestinal microbiota:from probiotics to gastrointestinal anti-infectious biotherapeutic agents. Clin Microbiol Rev. 2014;27(2):167-199.

6. Lee A, Lee YJ, Yoo HJ, et al. Consumption of Dairy Yogurt Containing Lactobacillus paracasei ssp. paracasei, Bifidobacterium animalis ssp. lactis and Heat-Treated Lactobacillus plantarum Improves Immune Function Including Natural Killer Cell Activity. Nutrients. 2017;9(6):558-566.

7. Bourgeois AL, Wierzba TF, Walker RI. Status of vaccine research and development for enterotoxigenic Escherichia coli. Vaccine. 2016;34(26):2880-2886.

8. Hartland EL, Leong JM. Enteropathogenic and enterohemorrhagic E. coli:ecology, pathogenesis, and evolution. Front Cell Infect Microbiol. 2013;3:15.

9. Sherman PM, Johnson-Henry CK, Yeung HP, et al. Probiotics Reduce Enterohemorrhagic Escherichia coli O157:H7- and Enteropathogenic E. coli O127:H6-Induced Changes in Polarized T84 Epithelial Cell Monolayers by Reducing Bacterial Adhesion and Cytoskeletal Rearrangements. Infect Immun. 2005;73(8):5183-5188.

10. Lan R, Alles MC, Donohoe K, et al. Molecular evolutionary relationships of enteroinvasive Escherichiacoli and Shigella spp. Infect Immun. 2004;72(9):5080-5088.

11. Nataro JP, Steiner T, Guerrant RL. Enteroaggregative Escherichia coli. Emerg Infect Dis. 1998;4(2):251-261.

12. Woo J, Ahn J. Probiotic-mediated competition, exclusion and displacement in biofilm formation by food-borne pathogens. Lett Appl Microbiol. 2013;56(4):307-313.

13. Abd El-Moez SI, Ahmed FY, Samy AA, et al. Probiotic Activity of $L$. acidophilus against Major Food-borne Pathogens Isolated from Broiler Carcasses. Nat Sci. 2010;8:69-78.

14. Kumar M, Dhaka P, Vijay D, et al. Antimicrobial effects of Lactobacillus plantarum and Lactobacillus acidophilus against multidrugresistant enteroaggregative Escherichia coli. Int $J$ Antimicrob Agents. 2016;48(3):265-270.

15. Jiang M, Zhang F, Wan C, Xiong Y, et al. Evaluation of probiotic properties of Lactobacillus plantarum WLPL04 isolated from human breast milk. J Dairy Sci. 2016;99(3):1736-1746.

16. Hirano J, Yoshida T, Sugiyama T, et al. The effect of Lactobacillus rhamnosus on enterohemorrhagic Escherichia coli infection of human intestinal cells in vitro. Microbiol Immunol. 2003;47(6):405-409.

17. Johnson-Henry KC, Donato KA, Shen-Tu G, et al. Lactobacillus rhamnosus strain GG prevents enterohemorrhagic Escherichia coli $\mathrm{O} 157: \mathrm{H} 7-$ induced changes in epithelial barrier function. Infect Immun. 2008;76(4):1340-1348.

18. Ogawa M, Shimizu K, Nomoto K, et al. Protective effect of Lactobacillus casei strain Shirota on Shiga toxin-producing Escherichia coli O157:H7 infection in infant rabbits. Infect Immun. 2001;69(2):1101-1108. 
19. Ferreira PC, Campos IB, Abe CM, et al. Immunization of mice with Lactobacillus casei expressing intimin fragments produces antibodies able to inhibit the adhesion of enteropathogenic Escherichia coli to cultivated epithelial cells. FEMS Immunol Med Microbiol. 2008;54(2):245-254.

20. Asahara T, Shimizu K, Nomoto K, et al. Probiotic bifidobacteria protect mice from lethal infection with Shiga toxin-producing Escherichia coli O157:H7. Infect Immun. 2004;72(4):2240-2247.
21. Hütt P, Shchepetova J, Lõivukene K, et al. Antagonistic activity of probiotic lactobacilli and bifidobacteria against entero- and uropathogens. J Appl Microbiol. 2006;100(6):1324-1332.

22. Shu Q, Lin H, Rutherfurd KJ, etal. Dietary Bifidobacterium lactis (HN019) enhances resistance to oral Salmonella typhimurium infection in mice. Microbiol Immunol. 2000;44(4):213-222.

23. Ashraf R, Shah NP. Immune system stimulation by probiotic microorganisms. Crit Rev Food Sci Nutr. 2014;54(7):938-956. 